\title{
Production of a biodegradable flocculant from cotton and evaluation of its performance in coagulation-flocculation of kaolin clay suspension: Optimization through response surface methodology (RSM)
}

Mohammad Nourani, Majid Baghdadi ${ }^{*}$, Mohammad Javan, Gholamreza Nabi Bidhendi

Department of Environmental Engineering, Graduate Faculty of Environment, University of Tehran, Tehran, Iran

Corresponding author: Majid Baghdadi

E-mail:_m.baghdadi@ut.ac.ir

Postal Address: Ghods Street, Enghelab Ave. Tehran, Iran

P.O.Box: 14155-6135

Phone: $\quad+982161113171$

Fax: $\quad+982166407719$ 


\begin{abstract}
Flocculants are widely used along with coagulant to increase the efficiency of coagulation-flocculation process for removal of colloidal particles from wastewaters. The most widely used flocculants are synthetic which have some problems such as non-biodegradability and release of toxic residual monomers. The main aim of this study is to evaluate the performance of cellulose sulfate (CS), which was synthesized through sulfonation of cotton, as a biodegradable and natural-based flocculant. Reaction time as an important factor in the sulfonation reaction was optimized for obtaining CS with high charge density and suitable fiber size. The sulfate content, charge density (CD) and morphology of each CS were determined to investigate and select the best CS for the flocculation process. A suspension containing kaolin clay was used as a colloidal test solution and coagulation was performed using alum (as a coagulant) and CS (as a flocculant). Optimization was performed using response surface methodology (RSM). The optimization results showed that CS had a good performance in turbidity reduction in coagulation-flocculation treatment and maximum turbidity reduction (98.9 \%) was obtained at alum dosage of $23.1 \mathrm{mg} / \mathrm{L}, \mathrm{CS}$ dosage of $7.2 \mathrm{mg} / \mathrm{L}$ and $\mathrm{pH}=6.2$. The results showed that the $\mathrm{pH}$ variation had insignificant effect on the $\mathrm{CS}$ dosage. Zeta potential measurements and microscopic photos of flocs confirm charge neutralization and bridge formation in flocculation using cellulose sulfate, respectively. Also, the total chemical consumption reduced compared with the use of alum alone. As a consequence, CS can be considered as a suitable alternative for conventional flocculants.
\end{abstract}

Keywords: Biodegradable; Flocculant; Turbidity removal; Cellulose sulfate; Coagulationflocculation; Response Surface Methodology 


\section{Introduction}

Coagulation-flocculation treatment is a suitable method for removing colloidal particles from municipal and industrial wastewater. Colloidal particles can be settled and removed easily by neutralization and making them closer. Recently, many studies have been conducted about using the coagulation-flocculation in industries, e.g., dairy [1], pulp [2], drinking water treatment [3], municipal wastewater treatment and pretreatment of landfill leachate [4].

In line with some studies, inorganic coagulants (mainly salts of multivalent metals) such as aluminum sulfate (alum) and ferric chloride have been used without assistance of flocculants [57]. Metal salts will hydrolyze rapidly in aqueous medium to form cationic species, and then they will be adsorbed by negatively charged colloidal particles. Therefore, simultaneous surface charge reduction and formation of micro-flocs will happen [3]. The micro-flocs are small and have low strength; therefore they will break down when subjected to physical forces. The low efficiency of the process and presence of metal residue in the supernatant indicate the necessity of using flocculants in coagulation-flocculation treatment [8].

There are a multitude of organic flocculants which most of them are synthetic, including poly-acrylamides, poly-acrylic acids, polystyrene sulfonic acids, and their derivatives. These flocculants are mostly linear water-soluble polymers with repeating units of various monomers, high molecular weight and can be ionic (addressed as polyelectrolyte) or non-ionic.

These flocculants have some problems such as non-biodegradability and release of residual monomers in water, which have adverse effects on the health $[9,10]$. So, using of the eco-friendly flocculants as alternative materials for conventional flocculants in water and Wastewater treatment is increasing $[11,12]$. 
Cellulose which is the most abundant and the most significant natural polymeric compound, has greatly influenced the human's life [13]. Because of biodegradability, availability, not-toxicity, high purity and low cost [14] it has been mostly considered in the water treatment process during recent years for instance in removal of dyes [15] and heavy metals [16, 17]. Recently, researchers have investigated the application of cellulose-based materials as a biodegradable flocculants in flocculation-coagulation process [3, 18-20]. The desired functionality can be introduced into the cellulose structure in order to prepare the most effective flocculant. Cellulose typically has a very large external surface area covered with hydroxyl groups (2-3 $\mathrm{mmol} / \mathrm{g})$. These unique properties provide an ideal platform for surface modification to develop a material with desired surface characteristics customized for a wide range of applications [21]. The flocs of iron hydroxide, which are formed during coagulation process, have positive charge. Therefore an anionic flocculant can interact with positively charged flocs to promote the formation of larger and more shear-resistant flocs as a consequence of bridge formation between destabilized particles. In this paper, cellulose was functionalized through sulfonation reaction to produce cellulose sulfate as an anionic flocculant.

Many factors are effective on the performance of the coagulation-flocculation process, such as dose of coagulant, dose of flocculant and initial pH. In many studies on the coagulationflocculation for turbidity removal, optimization was usually done by varying one factor while all other factors are constant $[3,18]$. This approach, consumes more time and energy and leads to inaccurate optimum because of ignoring the interaction of factors [22]. To overcome these problems, the response surface methodology (RSM) can be considered as an appropriate approach since including the impacts of individual factors and the ones of their interaction. RSM is consist of statistical and mathematical techniques and gives a comprehension of mathematical 
correlation between effective factors [4]. Using RSM leads to determine the optimum operational condition and a domain that satisfies the operating properties [23]. Recently, RSM methodology has been used to optimize coagulation-flocculation process for the wastewater treatment [2, 24].

The aim of this research is to study the ability and effectiveness of partially soluble anionic cellulose sulfate (CS) as a novel flocculant for removal of turbidity from aqueous medium containing suspended kaolin clay particles as a source of turbidity. For this purpose, four types of CS with different degrees of sulfonation, particle size and charge density were synthesized. The influences of alum, CS and solution $\mathrm{pH}$ on the coagulation-flocculation were studied by determining the residual turbidity of the settled suspension.

\section{Materials and methods}

\subsection{Materials}

The cellulose source for this study was hydrophilic natural cotton provided by Panberiz, Iran, company. Chlorosulfonic acid (CSA) and Dimethylformamide (DMF) were supplied by Merck (Darmstadt, Germany) and were used without further purification. Alum $\left(\mathrm{Al}_{2}\left(\mathrm{SO}_{4}\right)_{3} \cdot 18 \mathrm{H}_{2} \mathrm{O}\right)$ was employed as an inorganic coagulant agent and was also obtained from Merck. Commercial grade Kaolin clay (Superfine size of $11 \mu \mathrm{m}$ ) was procured from Kanipazhouh, Iran as a dry powder and its water slurry ( $\mathrm{pH}$ 7.4) was prepared in deionized water without any dispersing agent. The chemical composition of the kaolin sample was found to be $\mathrm{SiO}_{2} 60.17 \%, \mathrm{Al}_{2} \mathrm{O}_{3} 30.10 \%, \mathrm{Fe}_{2} \mathrm{O}_{3} 0.21 \%, \mathrm{MgO} 0.02 \%, \mathrm{CaO} 0.01 \%, \mathrm{~K}_{2} \mathrm{O} 0.13 \%, \mathrm{TiO}_{2} 0.03 \%$, with LOI of $6.31 \%$. The $\mathrm{pH}$ of the kaolin suspension was adjusted using $\mathrm{NaOH}$ and $\mathrm{HCl}$ purchased from Merck. 


\subsection{Synthesis of the cellulose sulfate (CS) flocculant}

Anionic CS with variable fiber size and charge density was synthesized by hydrolysis and sulfonation of cellulose with CSA in DMF. For this purpose, $3 \mathrm{~g}$ of hydrophilic natural cotton was immersed in $45 \mathrm{ml}$ of DMF in a dry and clean beaker for 15 minutes at ambient temperature. Then, $4.5 \mathrm{ml}$ of CSA was slowly added to $45 \mathrm{ml}$ of DMF in an ice bath. This process was followed by gradually adding this mixture to cotton immersed in DMF. Final mixture in the sealed glass container was shacked at a constant speed of $120 \mathrm{rpm}$ at ambient temperature. Sampling was performed at 45, 60, 75 and $90 \mathrm{~min}$. In order to separate DMF to reuse it, vacuum filtration was utilized. After filtration through a 1- $\mu \mathrm{m}$ membrane and washing with deionized water, the $\mathrm{pH}$ of obtained $\mathrm{CS}$ was neutralized by an adequate amount of sodium bicarbonate solution and then was filtered and washed again several times with deionized water. Schematic reaction for sulfonation of cellulose is shown in Fig. 1.

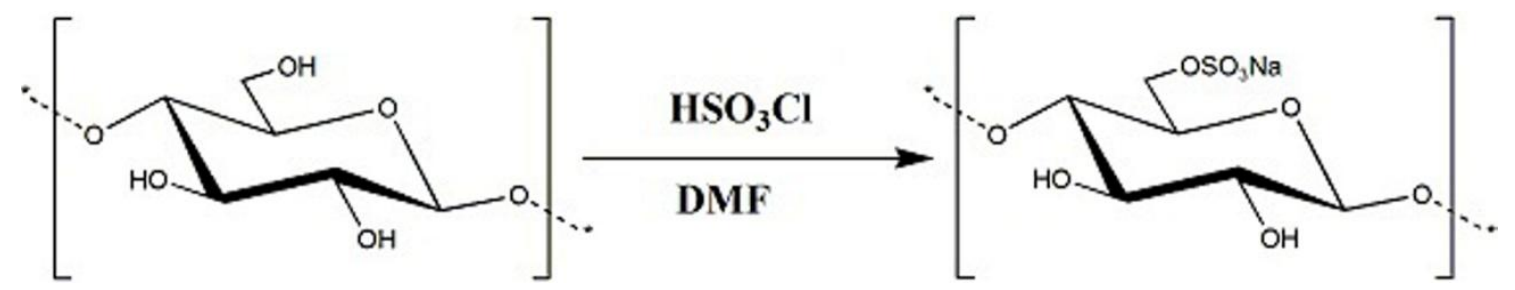

Fig. 1. Sulfonation of cellulose

\subsection{Characterization of CS flocculant}

Fourier transform infrared (FTIR) spectra of the cotton and CS were recorded using a Bruker FT-IR spectrometer. The amount of anionic groups of the CS was indirectly calculated from the sulfur content of the products, which was determined with an elemental analyzer (Thermo Finnigan, FlashEA 1112 series). Morphological studies of CSs were conducted by scanning electron microscope (SEM, model: HITACHI S-4160). The Malvern Zetasizer 3000 
was used to measure zeta potential of the kaolin clay suspension before and after adding alum. A six-beaker jar-test apparatus ( Zag-Chemi, Iran) was applied to perform coagulation-flocculation experiments. Residual turbidity of the supernatant was determined by HACH turbidimeter (model 2100Q01).

\subsection{Coagulation-flocculation experiments}

A standard jar-test was used to simulate coagulation-flocculation process and evaluate the flocculation performance of the CS. Kaolin suspension with a solid content of $5 \mathrm{~g} / \mathrm{L}$ was used as artificial turbidity. To perform experiments, pH of kaolin clay suspension (400 ml) was adjusted using solutions of $0.1 \mathrm{~mol} / \mathrm{L} \mathrm{HCl}$ and $0.1 \mathrm{~mol} / \mathrm{L} \mathrm{NaOH}$. The coagulation-flocculation process consisting of rapid mixing ( $3 \mathrm{~min}, 180 \mathrm{rpm}$ ), followed by slow mixing (15 min, $40 \mathrm{rpm}$ ) and settling at room temperature (30 min).

\subsection{Experimental design and data analysis}

The central composite design (CCD), which is one of the important designs of RSM, was selected for the optimization of the parameters. Since different variables are usually expressed in different units and/or have different limits of variation, the importance of their effects on the response can only be compared after they are coded. A total of 20 experiments according to a $2^{3}$ full factorial $\mathrm{CCD}$, consisting of eight factorial points (coded to the usual \pm 1 notation), six axial points $( \pm \alpha, 0,0),(0, \pm \alpha, 0),(0,0, \pm \alpha)$, and six replicates at the center points $(0,0,0)$ were conducted. The value of $\alpha$ for rotatability depends on the number of points in the factorial portion of the design, which is given in Eq. (1):

$\alpha=\left(N_{F}\right)^{1 / 4}$

where $N_{F}$ is the number of points in the cube portion of the design $\left(N_{F}=2^{k}, k\right.$ is the number of factors). Therefore, $\alpha$ is equal to $\left(2^{3}\right)^{1 / 4}=1.682$ according to Eq. (1). The relationship between 
the coded $\left(\mathrm{x}_{\mathrm{i}}\right)$ and actual $\left(\mathrm{X}_{\mathrm{i}}\right)$ values of factors and the ranges of levels are given in Table 1, in which the range of factors is expressed by the values of $X_{\min }($ minimum value of $X)$ and $X_{\max }$ (maximum value of $\mathrm{X}$ ).

Table 1 Relationship between the coded and actual values of factors and the levels of the variables tested in the central composite design.

\begin{tabular}{llccc}
\hline Code $\left(\mathrm{x}_{\mathrm{i}}\right)$ & Actual value of factor $\left(\mathrm{X}_{\mathrm{i}}\right)$ & $\begin{array}{c}\text { Coagulant dosage } \\
(\mathrm{mg} / \mathrm{L})\left(\mathrm{X}_{1}\right)\end{array}$ & $\begin{array}{c}\text { Flocculant dosage } \\
(\mathrm{mg} / \mathrm{L})\left(\mathrm{X}_{2}\right)\end{array}$ & $\begin{array}{c}\text { Initial } \mathrm{pH} \\
\left(\mathrm{X}_{3}\right)\end{array}$ \\
\hline$-\alpha$ & $\mathrm{X}_{\min }$ & 5.00 & 5.00 & 4.00 \\
-1 & $\frac{(\alpha-1) X_{\max }+(\alpha+1) X_{\min }}{2 \alpha}$ & 12.09 & 7.02 & 4.60 \\
0 & $\frac{X_{\max }+X_{\min }}{2}$ & 22.50 & 10.00 & 5.50 \\
1 & $\frac{(\alpha-1) X_{\min }+(\alpha+1) X_{\max }}{2 \alpha}$ & 32.91 & 12.97 & 6.39 \\
$+\alpha$ & $\mathrm{X}_{\max }$ & 40.00 & 15.00 & 7.00 \\
\hline
\end{tabular}

Turbidity reduction was selected as the dependent variable. The response variable was fitted by a second-order model in the form of quadratic polynomial equation:

$$
Y_{m}=b_{0}+\sum_{i=1}^{k} b_{i} X_{i}+\sum_{i=1}^{k} b_{i i} X_{i}^{2}+\sum_{i}^{i<j} \sum_{j} b_{i j} X_{i} X_{j}
$$

Where $\mathrm{Y}$ is the predicted response (turbidity removal efficiency), $\mathrm{b}_{0}$ the constant coefficient, $b_{i}$ the linear coefficients, $b_{i i}$ the quadratic coefficients, $b_{i j}$ the interaction coefficient and $x_{i}, x_{j}$ are the coded values of the variables. The actual and predicted values are presented in Table 2. The Design-Expert 9 software was used to design experiments and evaluate results. 
Table 2. Central composite design matrix with actual and predicted values

\begin{tabular}{|c|c|c|c|c|c|}
\hline \multirow[t]{2}{*}{ Run } & \multicolumn{3}{|c|}{ Coded values } & \multicolumn{2}{|c|}{ Turbidity removal (\%) } \\
\hline & Coagulant dosage $\left(\mathrm{X}_{1}\right)$ & Flocculant dosage $\left(\mathrm{X}_{2}\right)$ & $\mathrm{pH}\left(\mathrm{X}_{3}\right)$ & Actual & Predicted \\
\hline 1 & 1.68 & 0 & 0 & 97.42 & 97.12 \\
\hline 2 & 0 & 0 & 0 & 98.64 & 98.78 \\
\hline 3 & -1 & 1 & 1 & 96.42 & 96.41 \\
\hline 4 & 0 & 0 & 0 & 98.42 & 98.34 \\
\hline 5 & 0 & 1.68 & 0 & 97.78 & 97.78 \\
\hline 6 & 1 & 1 & 1 & 98.21 & 98.14 \\
\hline 7 & -1.68 & 0 & 0 & 93.71 & 93.83 \\
\hline 8 & 0 & 0 & 0 & 98.64 & 98.74 \\
\hline 9 & 0 & -1.68 & 0 & 97.71 & 98.01 \\
\hline 10 & 0 & 0 & 0 & 98.42 & 98.53 \\
\hline 11 & 0 & 0 & 0 & 98.42 & 98.33 \\
\hline 12 & -1 & -1 & -1 & 94.57 & 94.04 \\
\hline 13 & 0 & 0 & 1.68 & 98.07 & 98.21 \\
\hline 14 & 0 & 0 & 0 & 98.64 & 98.98 \\
\hline 15 & 1 & -1 & -1 & 96.21 & 96.01 \\
\hline 16 & 1 & 1 & -1 & 96.14 & 96.10 \\
\hline 17 & 0 & 0 & -1.68 & 94.07 & 93.81 \\
\hline 18 & 1 & -1 & 1 & 98.42 & 98.33 \\
\hline 19 & -1 & -1 & 1 & 96.57 & 96.92 \\
\hline 20 & -1 & 1 & -1 & 94.00 & 93.03 \\
\hline
\end{tabular}




\section{Results and discussion}

\subsection{Characteristics of CS}

FTIR spectra of source of cellulose (cotton) and CS are shown in Fig. 2. Sulfonation of cellulose was confirmed by the appearance of new IR bands at 997 and $1230 \mathrm{~cm}^{-1}$ which was respectively assigned to the symmetric and asymmetric stretching vibrations of sulfate group.

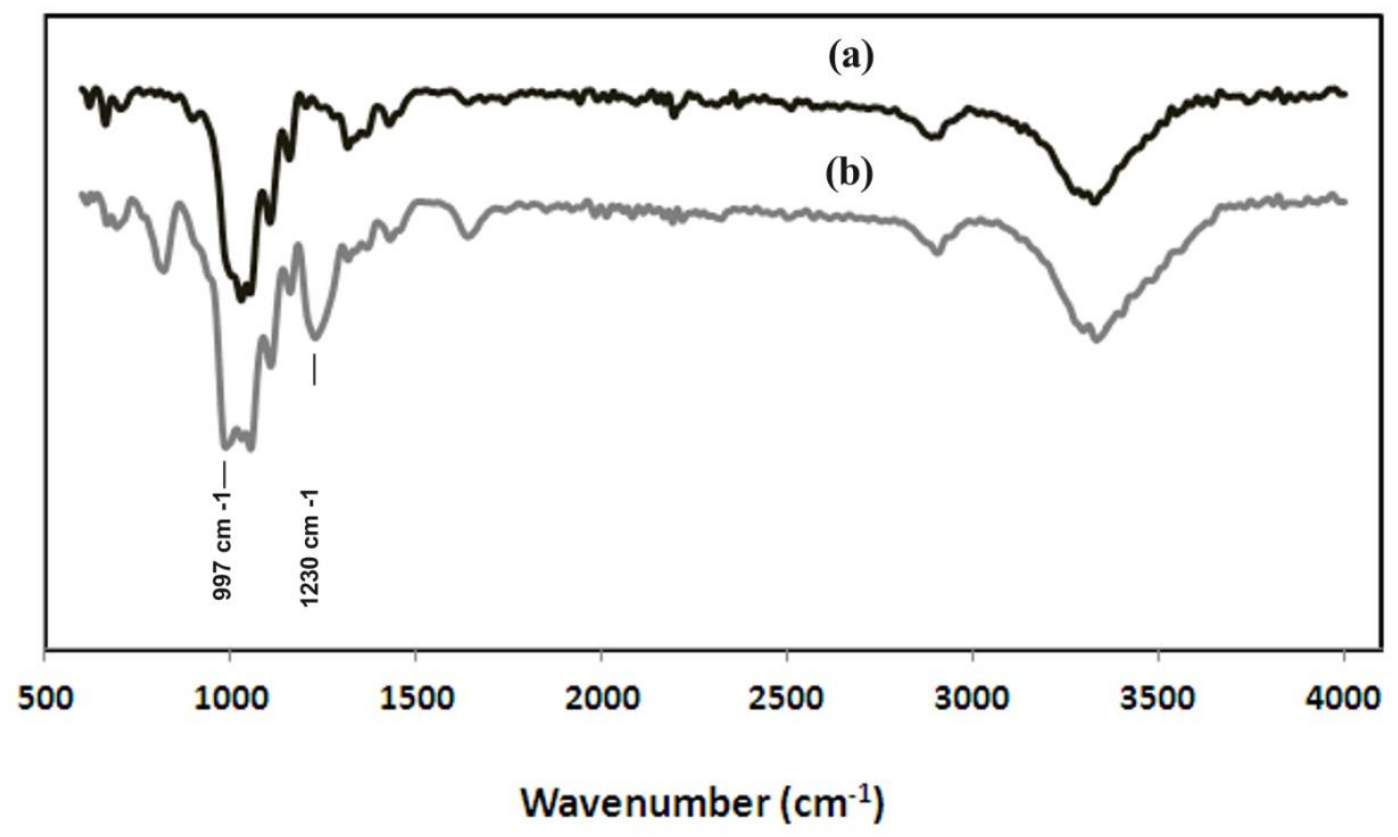

Fig. 2. FTIR spectra of (a) cellulose, (b) CS.

The sulfate content of anionic cellulose, which is representative the amount of anionic groups (charge density), was determined using an elemental analyzer. The effect of reaction time on the charge density is shown in Table 3. As can be seen, the charge density increased by increasing the time of reaction. 
Table 3. The effect of reaction time on the sulfur content and charge density

\begin{tabular}{cccc}
\hline Sample & Reaction time & Sulfur $(\%$ & Charge density \\
\hline CS I & 45 & 9.09 & 2.84 \\
CS II & 60 & 10.72 & 3.35 \\
CS III & 75 & 12.77 & 3.99 \\
CS IV & 90 & 13.57 & 4.24 \\
\hline
\end{tabular}

SEM images of cotton and CSs were investigated for understanding the surface morphology and fiber structure of synthetized CS. As demonstrated in Fig. 3, the sulfonation of cotton fibers led to significant change in surface morphology, which could be due to hydrolysis and destruction of cellulosic fiber structure in acidic medium. The row cotton had long, separate and rigid fibers, while by increasing the time of sulfonation, the surface of CSs become smoother. So that, after 60 minutes of sulfonation process, cotton fibers covert to smooth sheets. Our SEM images of CSs are similar to the results of sulfonated cellulose of hardwood kraft pulp, which was obtained by the sulfonation reaction with sodium bisulfite [25]. 

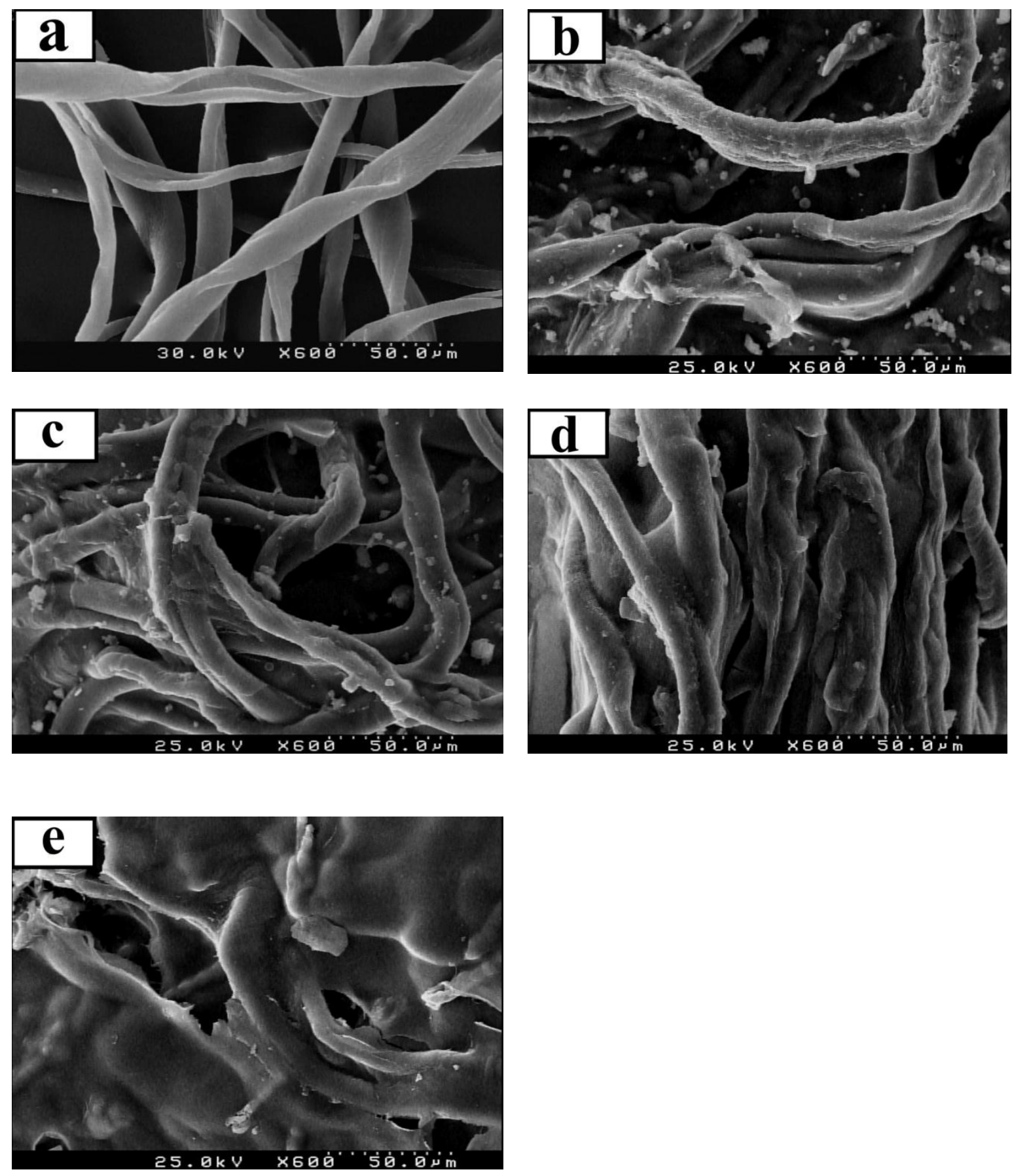

Fig. 3. SEM images of (a) cotton, (b) CS I, (c) CS II, (d) CS III, (e) CS IV 


\subsection{Comparison among flocculation efficiency of CSs}

In order to select the best CS as the flocculant, several jar tests were firstly performed without flocculent (CS) to choose an appropriate alum dosage at which the surface charge of kaolin clay has to be neutralized and aluminum hydroxide flocs does not have to be formed. As can be seen in Fig. 4, the turbidity of kaolin clay suspension $(5 \mathrm{~g} / \mathrm{L})$ decreased sharply after adding $20 \mathrm{mg} / \mathrm{L}$ alum and this decrease continues up to $250 \mathrm{mg} / \mathrm{L}$. These results indicate that the surface charge of kaolin clay has been neutralized at low dosage of alum by the hydrolyzed cationic aluminum species. In addition, the best coagulation performance of alum has been observed in the $\mathrm{pH}$ range of 4.5-6.5 [18]. Hence, the alum dosage of $20 \mathrm{mg} / \mathrm{L}$ and the $\mathrm{pH}$ of 5.5 were chosen for subsequent tests with CSs to make an appropriate comparison between their flocculation efficiency and sedimentation velocity.

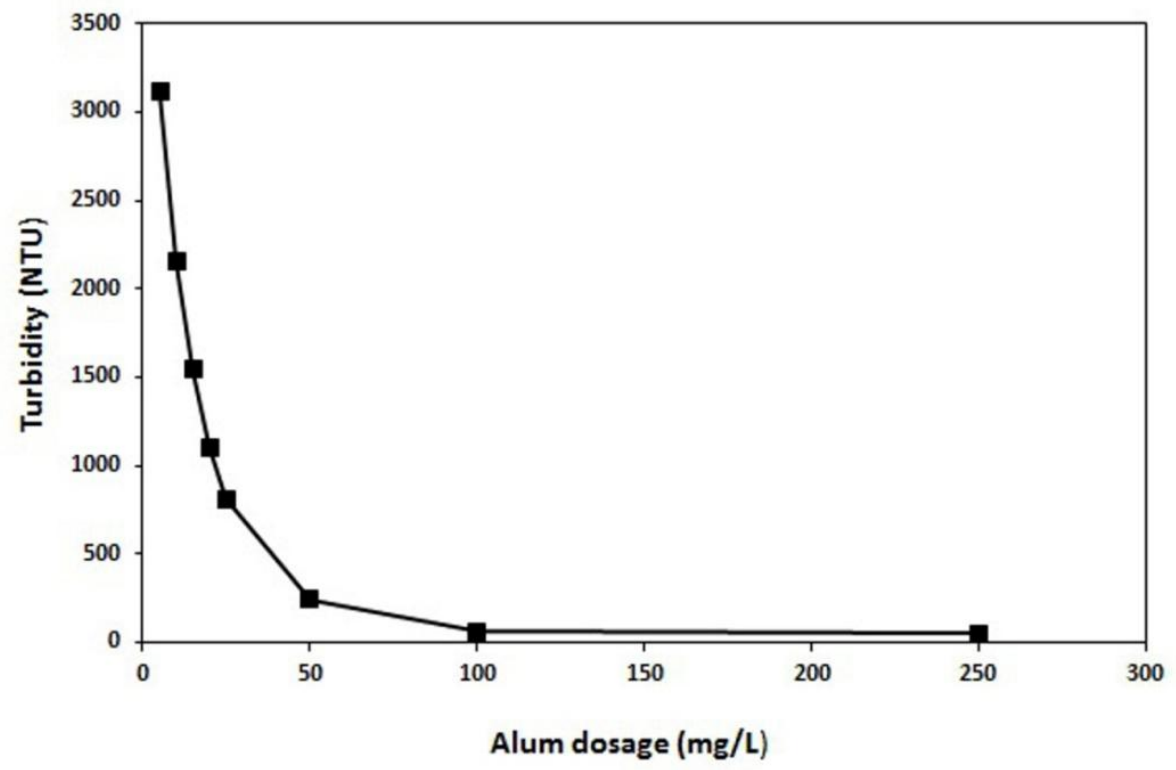

Fig. 4. Effect of alum dosage on the turbidity reduction of a kaolin suspension $(5 \mathrm{~g} / \mathrm{L})$.

pH: 5.5, Settling time: 30 min.

Several jar tests were performed to make an appropriate comparison between CSs flocculation efficiency and sedimentation velocity. The results of jar tests are presented in Fig. 5 
As it is shown, turbidity reduction and sedimentation velocity is higher for coagulationflocculation treatment with CS III. It is generally known that the most effective polymers for bridging are those possessing a high molecular weight [26], but the charge density can also have a considerable influence on bridging performance [27]. By increasing the time of reaction, from Cs I to CS IV, the charge density increases, but the fiber size decreases due to hydrolysis and destruction of cellulose fiber structure. The charge density of CS IV is greater than CS III, but its fiber length is smaller, therefore CS III shows better performance in turbidity reduction. Consequently, CS III was chosen for subsequent experiments.

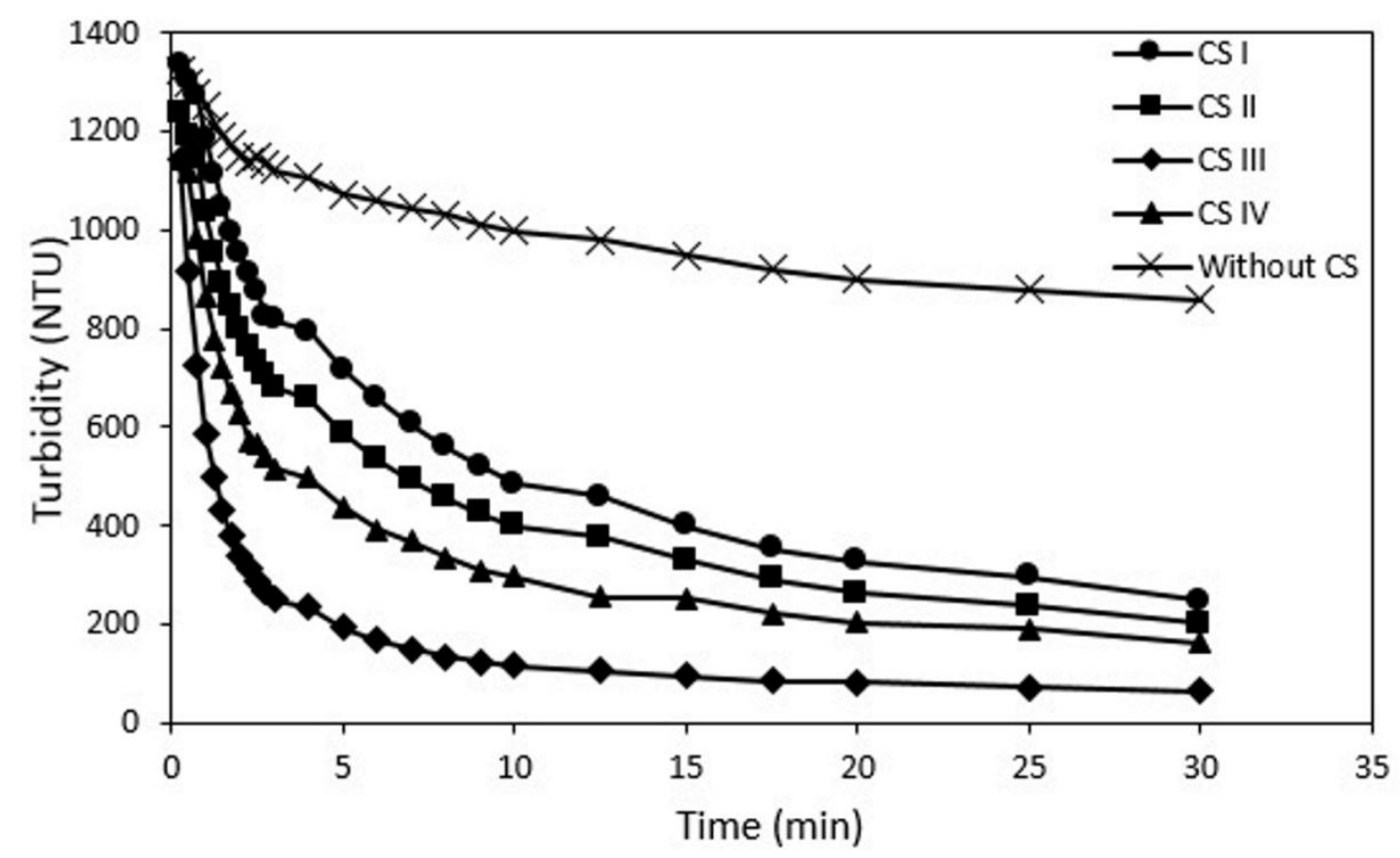

Fig. 5. Comparison among flocculation efficiency of CSs in coagulation-flocculation of kaolin suspension $(5 \mathrm{~g} / \mathrm{L})$. The alum dosage: $20 \mathrm{mg} / \mathrm{L}$, the CSs dosage: $10 \mathrm{mg} / \mathrm{L}$ and $\mathrm{pH}: 5.5$.

\subsection{Modeling and statistical Analysis}

The second-order polynomial equation was developed to correlate the turbidity removal percentage $(\mathrm{Y})$ as the function of coagulant dosage $\left(\mathrm{X}_{1}\right)$, flocculant dosage $\left(\mathrm{X}_{2}\right)$ and $\mathrm{pH}\left(\mathrm{X}_{3}\right)$. 
The following equation is a regression model with the experimental results:

$$
\begin{aligned}
Y= & 53.5305+0.4358 X_{1}+0.2068 X_{2}+12.4641 X_{3}+0.0048 X_{1} X_{2}+0.0083 X_{1} X_{3} \\
& +0.0495 X_{2} X_{3}-0.0095 X_{1}^{2}-0.0295 X_{2}^{2}-1.0743 X_{3}^{2}
\end{aligned}
$$

Statistical testing of the model was performed with the Fisher's statistical test for analysis of variance (ANOVA). The results of the ANOVA for turbidity reduction are shown in Table 4.

Table 4. Analysis of variance for turbidity removal

\begin{tabular}{lccccc}
\hline Source & Sum of squares & $\mathrm{dF}$ & Mean square & F-value & P-value, Prob $>$ F \\
\hline Model & 54.71 & 9 & 6.08 & 444.80 & $<0.0001$ \\
$\mathrm{X}_{1}$ & 12.50 & 1 & 12.50 & 914.64 & $<0.0001$ \\
$\mathrm{X}_{2}$ & $7.74 \times 10^{-4}$ & 1 & $7.74 \times 10^{-4}$ & 0.057 & 0.8172 \\
$\mathrm{X}_{3}$ & 15.74 & 1 & 15.74 & 1152.05 & $<0.0001$ \\
$\mathrm{X}_{1} \mathrm{X}_{2}$ & 0.13 & 1 & 0.13 & 9.47 & 0.0132 \\
$\mathrm{X}_{1} \mathrm{X}_{3}$ & 0.035 & 1 & 0.035 & 2.58 & 0.0240 \\
$\mathrm{X}_{2} \mathrm{X}_{3}$ & 0.10 & 1 & 0.10 & 7.34 & 0.1428 \\
$\mathrm{X}_{1}^{2}$ & 14.88 & 1 & 14.88 & 1088.85 & $<0.0001$ \\
$\mathrm{X}_{2}{ }^{2}$ & 0.95 & 1 & 0.95 & 69.83 & $<0.0001$ \\
$\mathrm{X}_{3}^{2}$ & 10.22 & 1 & 10.22 & 747.60 & $<0.0001$ \\
Residual & 0.12 & 9 & 0.014 & & 0.4928 \\
Lack of Fit & 0.054 & 4 & 0.014 & 0.98 & \\
Pure Error & 0.069 & 5 & & & \\
\hline
\end{tabular}

$\mathrm{R}^{2}=0.9955$, Adj $\mathrm{R}^{2}=0.0 .9872$ 
$P$-value determines whether $\mathrm{F}$ value is large enough to indicate statistical significance or not [28]. The p-values less than 0.05 indicate that the quadratic model terms are significant.

The model is highly significant with a very low probability value $(\mathrm{P}<0.0001)$. On the other hand, the lack of fit for this model ( $\mathrm{p}$-value $=0.4928$ ) is not significant. $A R^{2}$ value greater than 0.75 indicate the aptness of the model [29]. As can be seen, $R^{2}$ value is high $\left(R^{2}=0.9955\right)$ and reasonably agreed with the adjusted $\mathrm{R}^{2}$ value of 0.9872 . It means that the quadratic model fits the data and satisfactorily describe the system behavior within the range of operating factors [30]. The observed and predicted values by the quadratic model are shown in Table 2. The agreement between the turbidity removal by the model and the experimental data is very good. Adequate precision ratio obtained were greater than 4 , indicating that adequate signal for model could be used to negative the design space. The obtained ratio of 18.8 indicates an adequate signal.

\subsection{Three-dimension response surface and contour plots}

For a better explanation of the independent variables and their interactive effects on the turbidity reduction of kaolin clay suspension, 3D plots and its corresponding contour plots are represented in Fig. 6. To detect the interaction between factors, the shape of the contours can be used; indeed an elliptical shape in the contours shows significant interaction between factors, so, by using the response surface plot and the contour of each pair of independent factors, the optimal value of them and their interaction can be understood easily. The central ellipse in the contour diagram represents the highest predicted value for the response in the selected intervals

of factors. In fact, elliptical pattern represents some deep interaction between the independent variables. 
Fig. 6(a) shows that by increasing the alum and CS to a certain amount, turbidity removal reaches to a maximum and then decreases. This behavior suggests that floc breakup occurs because of charge reversal and dispersion when there is an extreme dosage of coagulant and flocculant [31, 32]. Zeta potential of kaolin suspension before and after adding alum was determined. The results showed that by increasing the alum dosage, the zeta potential decreased from $-15.5 \pm 2$ to $-1.2 \pm 0.5 \mathrm{mV}$ and after that a charge reversal was observed $(+3.5 \pm 1.2 \mathrm{mV})$ as a result of adsorption of excess $\mathrm{Fe}^{3+}$ on the surface of particles.

It can be seen in Fig. 6(a) that the turbidity reduction is more dependent on the coagulant dosage. This behavior suggests when the coagulant dosage is not sufficient, colloidal particles neutralization will not occur. Therefore, increasing or decreasing the flocculant dosage (CS) has no influence on the turbidity reduction. The $p$-value greater than 0.05 for flocculant dosage is also confirmed by the fact that while the surface charge of colloidal particles is not natural, CS dosage has insignificant effect on the turbidity removal.

Fig. 6(b) show that the turbidity removal decreases in the $\mathrm{pH}$ lower than 4.5 , because the isoelectric point (IEP) of kaolin clay is in the range of 4-5 [32] and at the $\mathrm{pH}$ lower than IEP, kaolin clay has positive surface charge. In addition, alum is a cationic coagulant and has no electrostatic interaction with kaolin clay that has positive surface charge. Also, in the $\mathrm{pH}$ above 6.5 , the turbidity removal decreases because anionic aluminum complexes are formed which can prevent floc formation [2].

The contour plot in Fig. 6 (c) is nearly linear and the $p$ value of $X_{2} X_{3}$ is 0.1428 ( $p$ value > 0.05), therefore the interaction between $\mathrm{CS}$ dosage and $\mathrm{pH}$ is insignificant. The sodium salt of cellulose sulfate is not classified as an acidic or basic flocculant; therefore it is anionic in the wide range of $\mathrm{pH}$ and variation of $\mathrm{pH}$ has no effect on its charge density, indicating insignificant 
interaction between $\mathrm{pH}$ and $\mathrm{CS}$ dosage. But the charge density of weak acidic flocculants such as carboxymethyl cellulose depends on the $\mathrm{pH}$ and it significantly decreases by decreasing the $\mathrm{pH}$. As a consequence, the low dosage of flocculant is required in the higher $\mathrm{pH}$, indicating significant interaction between $\mathrm{pH}$ and flocculant dosage.
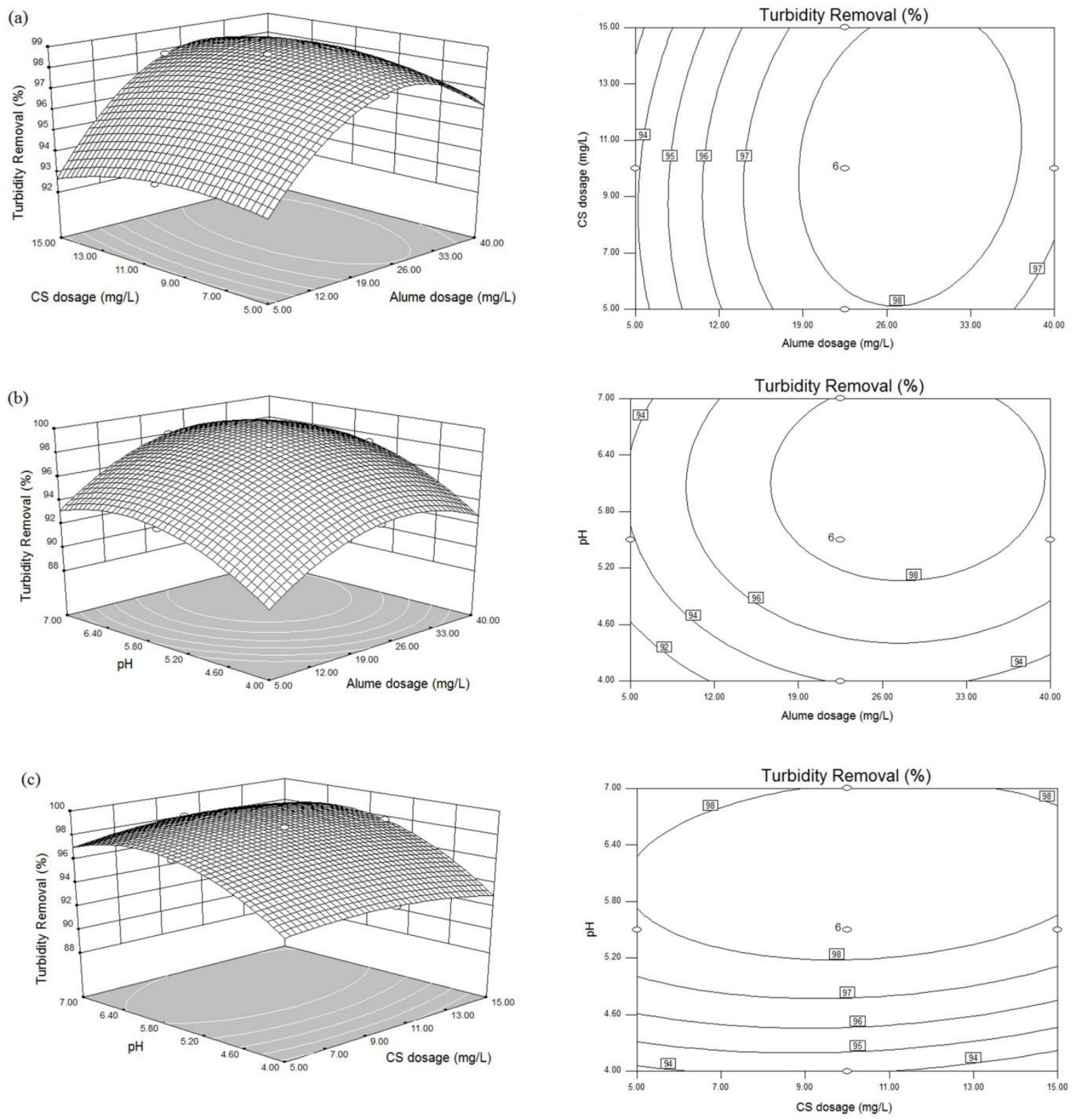
Fig 6. Surface plots and its corresponding contour plots of turbidity removal showing the effect of variables. (a)Alum dosage-CS dosage at $\mathrm{pH}$ of 5.5 (b) Alum dosage-pH at CS dosage of 10 $\mathrm{mg} / \mathrm{L}$ (c) pH-CS dosage at alum dosage of $22.5 \mathrm{mg} / \mathrm{L}$.

\subsection{Mechanism of flocculation with CS flocculant}

Kaolin particles are negatively charged in the neutral $\mathrm{pH}$ range ( $\mathrm{pH} 6$ to 8), causing their particles to remain in suspension without aggregating for long periods of time. Charge neutralization through adsorption of oppositely charged ions can be exploited to destabilize the colloidal suspensions. In order to investigate the neutralization of surface charge of kaolin particles with hydrolyzed cations of alum, zeta potential of suspension before and after adding alum was determined which the results showed reduction in zeta potential from $-15.5 \pm 2$ to $1.2 \pm 0.5 \mathrm{mV}$, respectively. After destabilization of kaolin particles, the cellulose sulfate was added to promote the formation of larger and more shear-resistant flocs as a consequence of bridge formation between destabilized particles. The microscopic photos of flocs in the absence and presence of CS are presented in Fig. 7. The larger flocs obtained in presence of CS confirms the bridge formation performance of cellulose sulfate. In addition to charge neutralization, sweep coagulation has an important role in turbidity reduction [33] through capture of small particles by large flocs in slow mixing. 


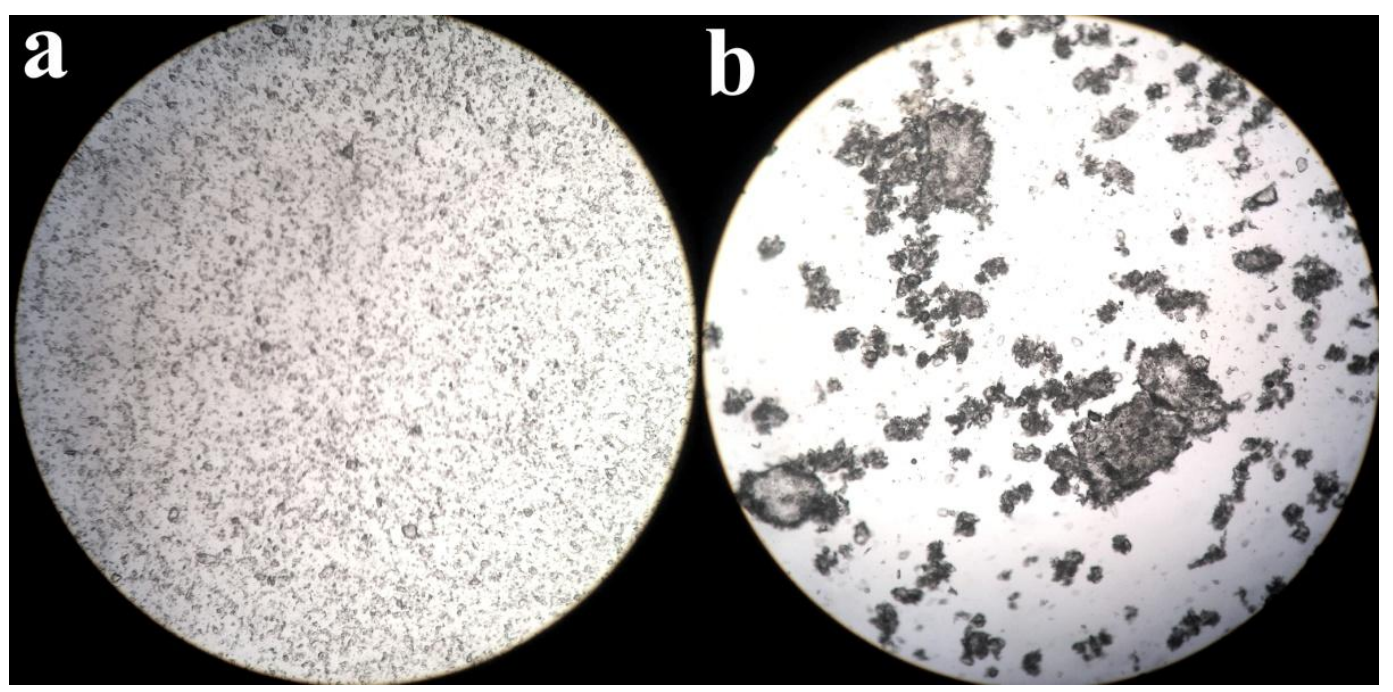

Fig. 7. Microscopic photos of flocs (a) in the absence of Cs and (b) in the presence of CS (Magnification: 10x)

\section{Conclusion}

A combined coagulation-flocculation treatment based on alum and a novel, partial soluble anionic cellulosic flocculant derived from cotton, CS, was found to be effective in removal of colloidal particles, as demonstrated using kaolin suspensions. A $2^{3}$ full factorial central composite design and response surface methodology was applied to overcome the limitations of classical methods and was successfully employed to obtain the optimum process conditions while the interactions between process variables were demonstrated. The optimize levels of alum dosage, CS dosage and $\mathrm{pH}$ for maximum turbidity reduction $(98.9 \%)$ were $23.1 \mathrm{mg} / \mathrm{L}, 7.2 \mathrm{mg} / \mathrm{L}$, 6.2 , respectively. In addition, the results revealed that the $\mathrm{pH}$ variation had minor effect on the CS dosage. Formation of larger flocs by bridging mechanism in presence of CS and sweep coagulation during sedimentation can lead to capture further microflocs. The combined treatment resulted in a lower residual turbidity of settled suspension with remarkably reduced total chemical consumption relative to coagulation with alum alone. 


\section{Acknowledgments}

We gratefully acknowledge financial support from Iran National Science Foundation (INSF) and

Nanotechnology Research Center of Graduate Faculty of Environment, University of Tehran.

\section{References}

[1] R. Ayeche, Treatment by Coagulation-Flocculation of Dairy Wastewater with the Residual Lime of National Algerian Industrial Gases Company (NIGC-Annaba), Energy Procedia 18 (2012) 147-156.

[2] J.-P. Wang, Y.-Z. Chen, Y. Wang, S.-J. Yuan, H.-Q. Yu, Optimization of the coagulation-flocculation process for pulp mill wastewater treatment using a combination of uniform design and response surface methodology, Water Research 45 (2011) 56335640 .

[3] T. Suopajärvi, H. Liimatainen, O. Hormi, J. Niinimäki, Coagulation-flocculation treatment of municipal wastewater based on anionized nanocelluloses, Chemical Engineering Journal 231 (2013) 59-67.

[4] X. Liu, X.-M. Li, Q. Yang, X. Yue, T.-T. Shen, W. Zheng, K. Luo, Y.-H. Sun, G.-M. Zeng, Landfill leachate pretreatment by coagulation-flocculation process using ironbased coagulants: optimization by response surface methodology, Chemical Engineering Journal 200 (2012) 39-51.

[5] H.-m. WANG, X.-b. MIN, L.-y. CHAI, Y.-d. SHU, Biological preparation and application of poly-ferric sulfate flocculant, Transactions of Nonferrous Metals Society of China 21 (2011) 2542-2547.

[6] J. Zhong, X. Sun, C. Wang, Treatment of oily wastewater produced from refinery processes using flocculation and ceramic membrane filtration, Separation and Purification Technology 32 (2003) 93-98.

[7] G. Dotto, G. Rosa, M. Moraes, R. Weska, L. Pinto, Treatment of chitin effluents by coagulation-flocculation with chitin and aluminum sulfate, Journal of Environmental Chemical Engineering 1 (2013) 50-55.

[8] C.S. Lee, J. Robinson, M.F. Chong, A review on application of flocculants in wastewater treatment, Process Safety and Environmental Protection 92 (2014) 489-508. 
[9] W. Brostow, H.H. Lobland, S. Pal, R.P. Singh, Polymeric flocculants for wastewater and industrial effluent treatment, Journal of Materials Education 31 (2009) 157-166.

[10] S. Chakrabarti, S. Banerjee, B. Chaudhuri, S. Bhattacharjee, B.K. Dutta, Application of biodegradable natural polymers for flocculated sedimentation of clay slurry, Bioresource technology 99 (2008) 3313-3317.

[11] H. Liimatainen, M. Visanko, J. Sirviö, O. Hormi, J. Niinimäki, Sulfonated cellulose nanofibrils obtained from wood pulp through regioselective oxidative bisulfite pretreatment, Cellulose 20 (2013) 741-749.

[12] H. Liimatainen, J. Sirviö, O. Sundman, M. Visanko, O. Hormi, J. Niinimäki, Flocculation performance of a cationic biopolymer derived from a cellulosic source in mild aqueous solution, Bioresource technology 102 (2011) 9626-9632.

[13] A.W. Carpenter, C.-F. de Lannoy, M.R. Wiesner, Cellulose Nanomaterials in Water Treatment Technologies, Environmental science \& technology 49 (2015) 5277-5287.

[14] V.K. Gupta, S. Agarwal, P. Singh, D. Pathania, Acrylic acid grafted cellulosic Luffa cylindrical fiber for the removal of dye and metal ions, Carbohydrate polymers 98 (2013) 1214-1221.

[15] R. Batmaz, N. Mohammed, M. Zaman, G. Minhas, R.M. Berry, K.C. Tam, Cellulose nanocrystals as promising adsorbents for the removal of cationic dyes, Cellulose 21 (2014) 1655-1665.

[16] S. Hokkanen, E. Repo, S. Lou, M. Sillanpää, Removal of arsenic (V) by magnetic nanoparticle activated microfibrillated cellulose, Chemical Engineering Journal 260 (2015) 886-894.

[17] H.-X. Zhu, X.-J. Cao, Y.-C. He, Q.-P. Kong, H. He, J. Wang, Removal of Cu 2+ from aqueous solutions by the novel modified bagasse pulp cellulose: Kinetics, isotherm and mechanism, Carbohydrate Polymers 129 (2015) 115-126.

[18] H. Liimatainen, J. Sirviö, O. Sundman, O. Hormi, J. Niinimäki, Use of nanoparticular and soluble anionic celluloses in coagulation-flocculation treatment of kaolin suspension, water research 46 (2012) 2159-2166.

[19] J. Sirviö, A. Honka, H. Liimatainen, J. Niinimäki, O. Hormi, Synthesis of highly cationic water-soluble cellulose derivative and its potential as novel biopolymeric flocculation agent, Carbohydrate Polymers 86 (2011) 266-270. 
[20] T. Suopajärvi, E. Koivuranta, H. Liimatainen, J. Niinimäki, Flocculation of municipal wastewaters with anionic nanocelluloses: Influence of nanocellulose characteristics on floc morphology and strength, Journal of Environmental Chemical Engineering 2 (2014) 2005-2012.

[21] D. Vandamme, S. Eyley, G. Van den Mooter, K. Muylaert, W. Thielemans, Highly charged cellulose-based nanocrystals as flocculants for harvesting Chlorella vulgaris, Bioresource Technology 194 (2015) 270-275.

[22] R.L. Mason, R.F. Gunst, J.L. Hess, Statistical design and analysis of experiments: with applications to engineering and science, John Wiley \& Sons2003.

[23] S.S. Moghaddam, M.A. Moghaddam, M. Arami, Coagulation/flocculation process for dye removal using sludge from water treatment plant: optimization through response surface methodology, Journal of hazardous materials 175 (2010) 651-657.

[24] Y. Yang, Y. Li, Y.-m. Zhang, D.-w. Liang, Applying hybrid coagulants and polyacrylamide flocculants in the treatment of high-phosphorus hematite flotation wastewater (HHFW): Optimization through response surface methodology, Separation and Purification Technology 76 (2010) 72-78.

[25] D. Rajalaxmi, N. Jiang, G. Leslie, A.J. Ragauskas, Synthesis of novel water-soluble sulfonated cellulose, Carbohydrate research 345 (2010) 284-290.

[26] L. Ghimici, M. Nichifor, Flocculation by cationic amphiphilic polyelectrolyte: Relating efficiency with the association of polyelectrolyte in the initial solution, Colloids and Surfaces A: Physicochemical and Engineering Aspects 415 (2012) 142-147.

[27] L. Ghimici, M. Nichifor, Novel biodegradable flocculanting agents based on cationic amphiphilic polysaccharides, Bioresource technology 101 (2010) 8549-8554.

[28] R. Sridhar, V. Sivakumar, J.P. Maran, K. Thirugnanasambandham, Influence of operating parameters on treatment of egg processing effluent by electrocoagulation process, International Journal of Environmental Science and Technology 11 (2014) 16191630.

[29] S.S. Naik, Y.P. Setty, Optimization of parameters using response surface methodology and genetic algorithm for biological denitrification of wastewater, International Journal of Environmental Science and Technology 11 (2014) 823-830.

[30] A. Bhanarkar, R. Gupta, R. Biniwale, S. Tamhane, Nitric oxide absorption by hydrogen peroxide in airlift reactor: a study using response surface methodology, International Journal of Environmental Science and Technology 11 (2014) 1537-1548. 
[31] Y. Wang, K. Chen, L. Mo, J. Li, J. Xu, Optimization of coagulation-flocculation process for papermaking-reconstituted tobacco slice wastewater treatment using response surface methodology, Journal of Industrial and Engineering Chemistry 20 (2014) 391396.

[32] Ž. Dohnalová, L. Svoboda, P. Šulcová, Characterization of kaolin dispersion using acoustic and electroacoustic spectroscopy, Journal of Mining and Metallurgy, Section B: Metallurgy 44 (2008) 63-72.

[33] J. Gregory, C.R. O'Melia, Fundamentals of flocculation, Critical Reviews in Environmental Science and Technology 19 (1989) 185-230. 